\title{
BreastCare
}

\section{Alleviating the Breast Cancer Experience: A Plea for Psycho-Oncology}

\author{
Kerstin Hermelink $^{\mathrm{a}} \quad$ Ute Berndt $^{\mathrm{b}}$ Renate Haidinger ${ }^{\mathrm{c}}$ \\ a Breast Center, Department of Gynecology and Obstetrics, CCC University Hospital of Munich, Germany; \\ ${ }^{b}$ Department of Gynaecology, Martin-Luther-Universitaet Halle-Wittenberg, Halle (Saale), Germany; \\ ${ }^{\mathrm{c}}$ Brustkrebs Deutschland e.V., Munich, Germany
}

In public perception, breast cancer seems to increasingly have become a manageable disease - to the point that breast cancer patients are sometimes faced with minimization of their condition, expressed in remarks like, 'Don't be upset - it's only breast cancer.' Indeed, prospects have considerably brightened for breast cancer patients. Nowadays, the vast majority of them will never develop metastatic disease, and even for those who do there is often some reasonable hope for many more years of life. Nevertheless, in a recently published large-scale investigation, mental disorders were found to be present in $42 \%$ of breast cancer patients. No other group of cancer patients showed an equally high rate of psychological morbidity [1]. Why do breast cancer patients experience exceptionally high levels of distress, despite a generally favorable prognosis?

Even with enormous advances in diagnosis and treatment of breast cancer, no affected woman can be sure of permanent cure. Disease progression and its dire consequences remain a constant threat for decades after primary diagnosis. Further, the treatments that are so efficaciously saving lives are at the same time not very successful at preserving the patients' physical, functional, and psychosocial wellbeing; thus, they come at high costs for the patients [2]. In particular, breast cancer therapy is a massive attack on a woman's female integrity, affecting nearly all key aspects of femininity; namely sexuality, child bearing, ability to breastfeed, and feminine attraction. Both chemotherapy and endocrine therapy have long-lasting or even permanent consequences for sexual functioning [3]. Chemotherapy threatens to destroy a patient's fertility; endocrine therapy deprives her of the actions of estrogens and may force her to delay child bearing for as long as 10 years - which mostly implies forgoing it. Further, chemotherapy inevitably damages a woman's good looks for the better part of a year and bereaves her of a part of her body that is precious to most women, her hair. A considerable number of breast cancer patients still have to undergo mastectomy. This may not only result in disfigurement but also means losing a highly valued organ that plays an important part in sexual activity and - for young women - in child raising. Sophisticated oncoplastic surgery techniques are now available that potentially achieve satisfactory cosmesis; sexual and - probably much less important but not negligible - lactational capacities of the breast, however, are irrevocably lost. Surgery and radiation of the axilla, still necessary in many cases, bear a strong risk of lymphedema that may lead to considerable disfigurement as well. In addition to these harms to femininity, breast cancer therapy exposes many patients to appallingly aversive states that are inadequately labeled as nausea and vomiting, and there is a plethora of other deleterious side effects, several of them painful and even disabling, e.g. peripheral neuropathy. Some breast cancer patients navigate these tribulations with stupefying resilience - many others, understandably, are overwhelmed by them.

This not only burdens the patients themselves but also their families. As psycho-oncologists (K.H., U.B.) and patient advocates (R.H.), we are sometimes told what it was like to be a child in a family affected by breast cancer. Some of the stories that are recounted to us are heartbreaking, and they have repercussions throughout adult life, like that of a young patient who consulted one of us because she tended to totally relinquish her own wishes and needs, whereas she anxiously sought to satisfy those of the persons close to her. She reported that her mother had been diagnosed with breast cancer when she herself was a schoolchild. She tried whatever she could to relieve her mother's burden and was happy when her mother was at home and did not cry. The family did not talk about the situation at all. Her father, her little brother, she herself and her mother - all had needed help but none of them received it. When her mother finally died, her devastated father left his adolescent children to return only years later.

Psycho-oncology was still in its infancy when this happened some 30 years ago, while it is now firmly established as an integral, indispensable part of breast cancer therapy. Gratifyingly, guide-

\section{KARGER}

Fax +497614520714
() 2015 S. Karger GmbH, Freiburg

$1661-3791 / 15 / 0102-0082 \$ 39.50 / 0$
Dr. Kerstin Hermelink

Klinik und Poliklinik für Frauenheilkunde und Geburtshilfe,

Klinikum der Universität München, Campus Großhadern

Marchioninistr. 15, 81377 München, Germany

Kerstin.Hermelink@med.uni-muenchen.de 
lines are progressively emerging that set standards for identification and treatment of distressed cancer patients, such as the new German psycho-oncology guideline that is described in the editorial by Joachim Weis in this issue [4]. Certainly, psycho-oncology cannot prevent all heartbreak; however, a recent meta-analysis found evidence that psycho-oncologic interventions have sustained small-to-medium effects on emotional distress and quality of life in cancer patients in general. In highly distressed patients, the effects were found to be large [5].
In the issue that is accompanied by this editorial, Susanne Singer and her colleagues report on breast cancer patients' fears of different treatments as assessed in the large-scale BRENDA II study [6]. Tanja Zimmermann looks into the consequences of breast cancer for both partners in an intimate relationship and delineates interventions for couples [7]. Valerie A. Jenkins and Lesley J. Fallowfield have investigated the reasons why women with breast cancer consent or decline to participate in randomized clinical trials - with surprising results [8].

\section{References}

1 Mehnert A, Brähler E, Faller H, et al.: Four-week prevalence of mental disorders in patients with cancer across major tumor entities. J Clin Oncol 2014;32: 3540-3546.

2 Fallowfield L, Jenkins V: Psychosocial/survivorship issues in breast cancer: are we doing better? J Natl Cancer Inst 2015;107:335.

3 Krychman M, Millheiser LS: Sexual health issues in women with cancer. J Sex Med 2013;10 Suppl 1:5-15.
Weis J: Psychosocial care for cancer patients. Breast Care 2015;DOI:10.1159/000381969.

5 Faller H, Schuler M, Richard M, et al.: Effects of psychooncologic interventions on emotional distress and quality of life in adult patients with cancer: systematic review and meta-analysis. J Clin Oncol 2013, 31:782-793.

-6 Singer S, Blettner M, Kreienberg R, et al.: Breast cancer patients' fear of treatment: results from the multi-centre longitudinal study BRENDA II. Breast Care 2015; DOI:10.1159/000381933.
Zimmermann T: Intimate relationships affected by breast cancer: interventions for couples. Breast Care 2015;DOI:10.1159/000381966.

8 Jenkins VA, Fallowfield LJ: For the benefit of others: reasons why women with breast cancer participate in RCTs. Breast Care 2015;DOI:10.1159/000376563. 